\title{
A Potential Pitfall in Parathyroid Scintigraphy: Description of A Rare Case
}

\author{
Antonella Francini ${ }^{\mathrm{a}}$, Ignazio Emmolo ${ }^{\mathrm{b}}$, Giuseppe Forte ${ }^{\mathrm{c}}$, Franco Acchiardi ${ }^{\mathrm{a}}$, \\ Stephane Chauvie ${ }^{\mathrm{a}}$ e, Giorgio Borretta $^{\mathrm{d}}$, Alberto Biggi ${ }^{\mathrm{a}}$
}

\begin{abstract}
A 32 years old male patient after admission at our center was diagnosed as a primary hyperparathyroidism. Diagnostic imaging provided two different alternatives for the cause of the disease: an adenoma/carcinoma of the parathyroid gland or a hyper functioning thyroid node. The histology after surgical excision proved to be an adenoma of the parathyroid gland. This case report demonstrated this rare behavior for parathyroid scintigraphy in which parathyroid adenoma concentrates $\mathrm{Tc}^{99 \mathrm{~m}}$-sestaMIBI in the early and late phase but also $\mathrm{Tc}^{99 \mathrm{~m}}$-pertechnetate.
\end{abstract}

Keywords: Parathyroid; Scintigraphy

\section{Case Report}

A 32 years old Caucasian male after admission at our centre was diagnosed as a primary hyperparathyroidism (PHPT). He worked as a teacher, weighted $75 \mathrm{~kg}$ and was 1.7 meters tall. The patient was non-smoking and declared of not drinking alcohol or using drugs. He reported to have been in general good condition except for a renal colic occurred seven months before. He presented with intense epigastralgia.

Manuscript accepted for publication August 18, 2010

${ }^{a}$ Nuclear Medicine Service, Santa Croce e Carle Hospital, Cuneo, Italy

${ }^{\mathrm{b}}$ Casa di cura citta di Bra, Bra, Italy

${ }^{c}$ Pathology Service, Santa Croce e Carle Hospital, Cuneo, Italy

${ }^{\mathrm{d}}$ Endocrinology and Metabolism Service, Santa Croce e Carle Hospital,

Cuneo, Italy

${ }^{\mathrm{e}}$ Corresponding author: Nuclear Medicine Service, Santa Croce e Carle

Hospital, via Coppino 26, I-12100, Cuneo, Italy.

Email: chauvie.s@ospedale.cuneo.it

doi:10.4021/jmc39w
Blood analysis revealed severe hypercalcaemia $(14.8 \mathrm{mg} / \mathrm{dl})$ and elevated parathyroid hormone levels $(665 \mathrm{pg} / \mathrm{ml})$. Renal and thyroid functions were normal. Bone Mineral Density values were low ( $\mathrm{T}$ score: lumbar spine $=-2.39 \mathrm{SD}$, femur $=-2.12 \mathrm{SD}$, forearm $=-2.16 \mathrm{SD}$ ). No urinary lithiasis was documented. MEN syndrome was excluded. Patient was addressed to diagnostic imaging to eventually localize hyper functional parathyroid tissue.

\section{Diagnostic imaging}

The patient was initially studied with ultrasonography (Fig. 1) that demonstrated a large node contiguous to the left thyroid lobe. The patient was addressed to our nuclear medicine protocol that consists of a thyroid scintigraphy followed by a parathyroid one. Thyroid scintigraphy using $\mathrm{Tc}^{99 \mathrm{~m}}$-pertechnetate (Fig. 2) showed a large intense uptake of tracer in the left lower pole of the thyroid and parathyroid scintigraphy using $\mathrm{Tc}^{99 \mathrm{~m}}$-sestaMIBI (Fig. 3) revealed an intense and persistent uptake on the tracer in the same region.

Usually, parathyroid adenoma shows an area of early and delay hyperconcentration of $\mathrm{Tc}^{99 \mathrm{~m}}$-sestaMIBI, while it does not concentrate $\mathrm{Tc}^{99 \mathrm{~m}}$-pertechnethate. Sometimes may show an uptake of $\mathrm{Tc}^{99 \mathrm{~m}}$-sestaMIBI only in the early or only in the late images (Table 1). But, hardly ever, the parathyroid adenoma accumulates $\mathrm{Tc}^{99 \mathrm{~m}}$-pertechnetate [2].

\section{Surgery}

To correct for the severe PHPT, the patient underwent surgery with the thought that the large node could be either an adenoma/carcinoma of the parathyroid gland or a hyper functioning thyroid node.

During the surgical intervention the large node shown in imaging was situated in the posterior-inferior part of the thyroid left lobe (Fig. 4). The excised node was identified by the histological frozen section as pathological parathyroid tissue (ptosic superior adenoma) (Fig. 5).

The left inferior parathyroid, which was normal both in size and appearance (Fig. 6), was not excised also because PTH presented a remarkable drop during surgical procedure (from $484 \mathrm{pg} / \mathrm{ml}$ to $51 \mathrm{pg} / \mathrm{ml}$ ). 


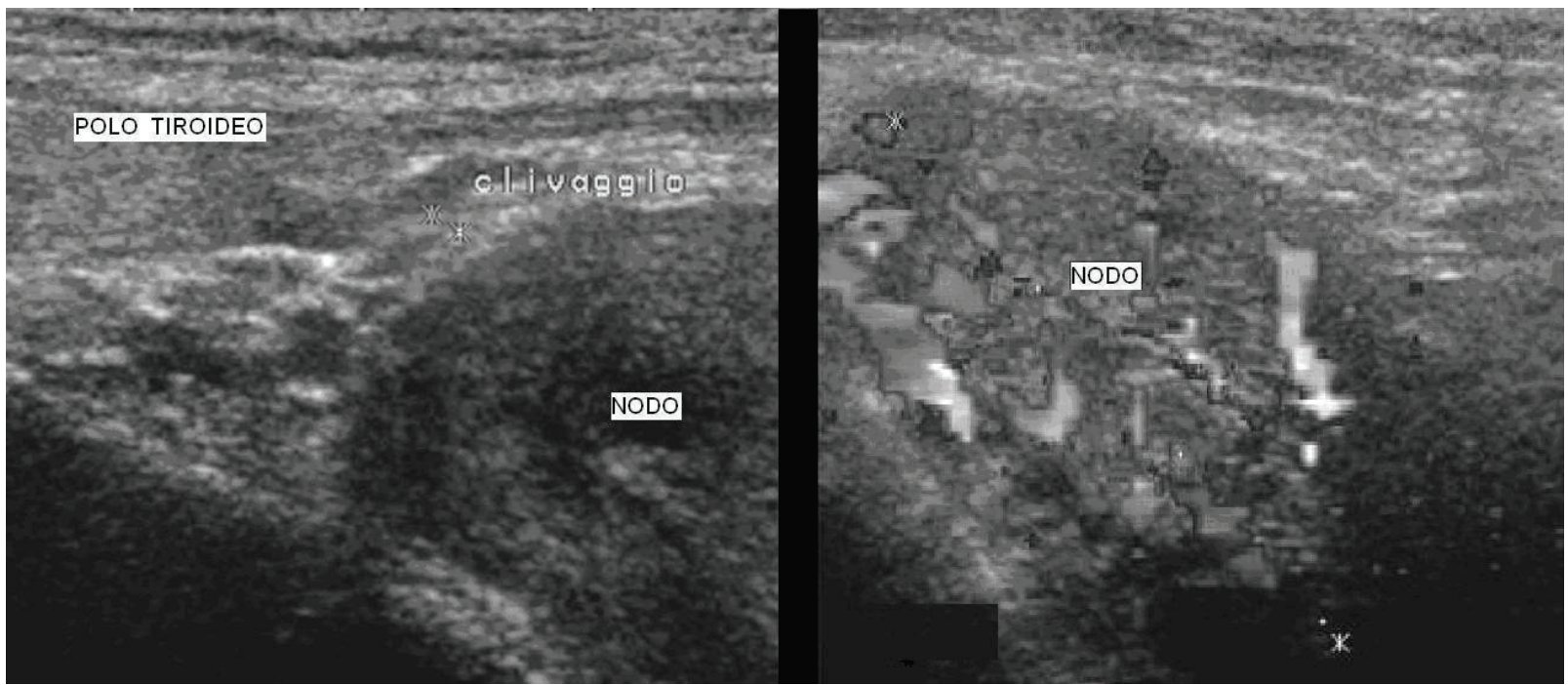

Figure 1. Ultrasonography. Ultrasound scan showed a large solid $(41 \times 31 \times 24 \mathrm{~mm})$ hypo-echogen node contiguous to the left thyroid lobe. On the right picture we could observe the high intranodal vascularisation.

The final histological examination confirmed the diagnosis of adenoma of the parathyroid gland. The performed tissue was tested by immunohistochemical analysis with monoclonal antibodies for thyreoglobuline and TTF-1 and resulted negative. The cellular proliferation evaluated by monoclonal antibody Ki-67 was less then 2-3\%.

The patient was behaving well at eight months followup.

\section{Discussion}

The new possibility offered by the mininvasive parathyroid surgery greatly advantages by the possibility of localizing the adenoma with nuclear and ultrasound imaging. There is

Table 1. Different Patterns of Radiopharmaceuticals Uptake

\begin{tabular}{cccc}
\hline \multirow{2}{*}{ Pattern } & \multicolumn{3}{c}{ Uptake in Parathyroid Adenoma } \\
& $\mathrm{Tc}^{99 \mathrm{~m}}$-SestaMIBI & $\mathrm{Tc}^{99 \mathrm{~m}}$-pertechnetate \\
& Early & Late & \\
\hline 1 (usual) & Yes & Yes & No \\
2 (not frequent) & Yes & No & No \\
3 (not frequent) & No & Yes & No \\
4 (hardly ever) & Yes & Yes & Yes \\
\hline
\end{tabular}

general consensus that the $\mathrm{Tc}^{99 \mathrm{~m}}$-sestaMIBI is the best way to localize preoperatively the hyper secreting parathyroid tissue. A meta-analysis of the sensitivity and specificity of this

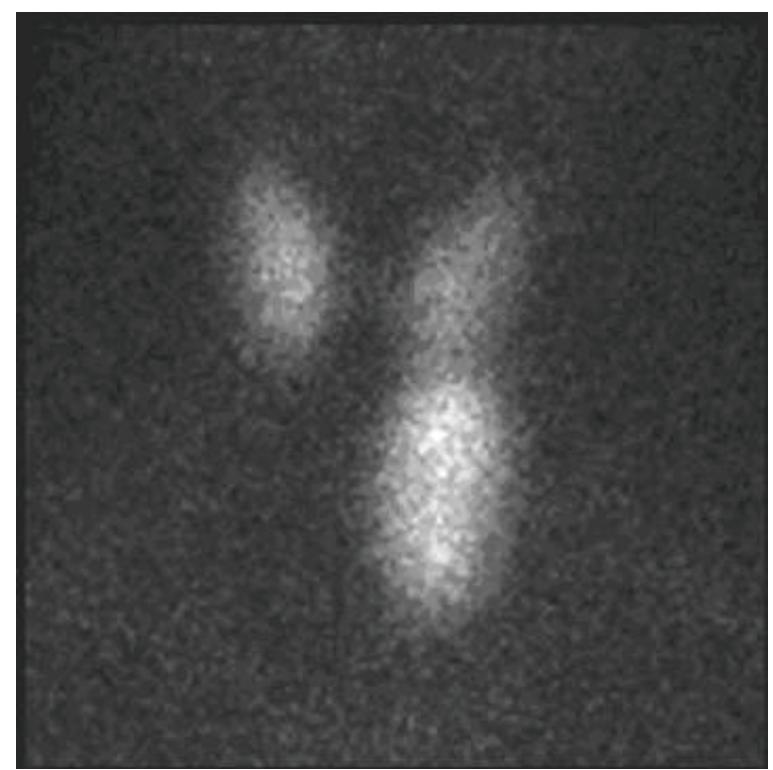

Figure 2. Thyroid scintigraphy. $\mathrm{Tc}^{99 \mathrm{~m}}$-pertechnetate, that mimics iodine, is rapidly concentrated in the thyroid but conversely to iodine is quickly released from the thyroid with a half life of about one hour. While $\mathrm{Tc}^{99 \mathrm{~m}}$-pertechnetate accumulates in the healthy thyroid it usually does not accumulate in the parathyroid [1]. The thyroid study was obtained 15 minutes after the administration of $74 \mathrm{MBq}$ of $\mathrm{Tc}^{99 \mathrm{~m}}$-pertechentate. All the images were obtained with a Millennium VG gamma camera (GE Healthcare, Wisconsin, USA) using a LEHR collimator. Thyroid scintigraphy using $\mathrm{Tc}^{99 \mathrm{~m}}$-pertechnetate showed a large intense uptake of tracer in the left lower pole of the thyroid. 


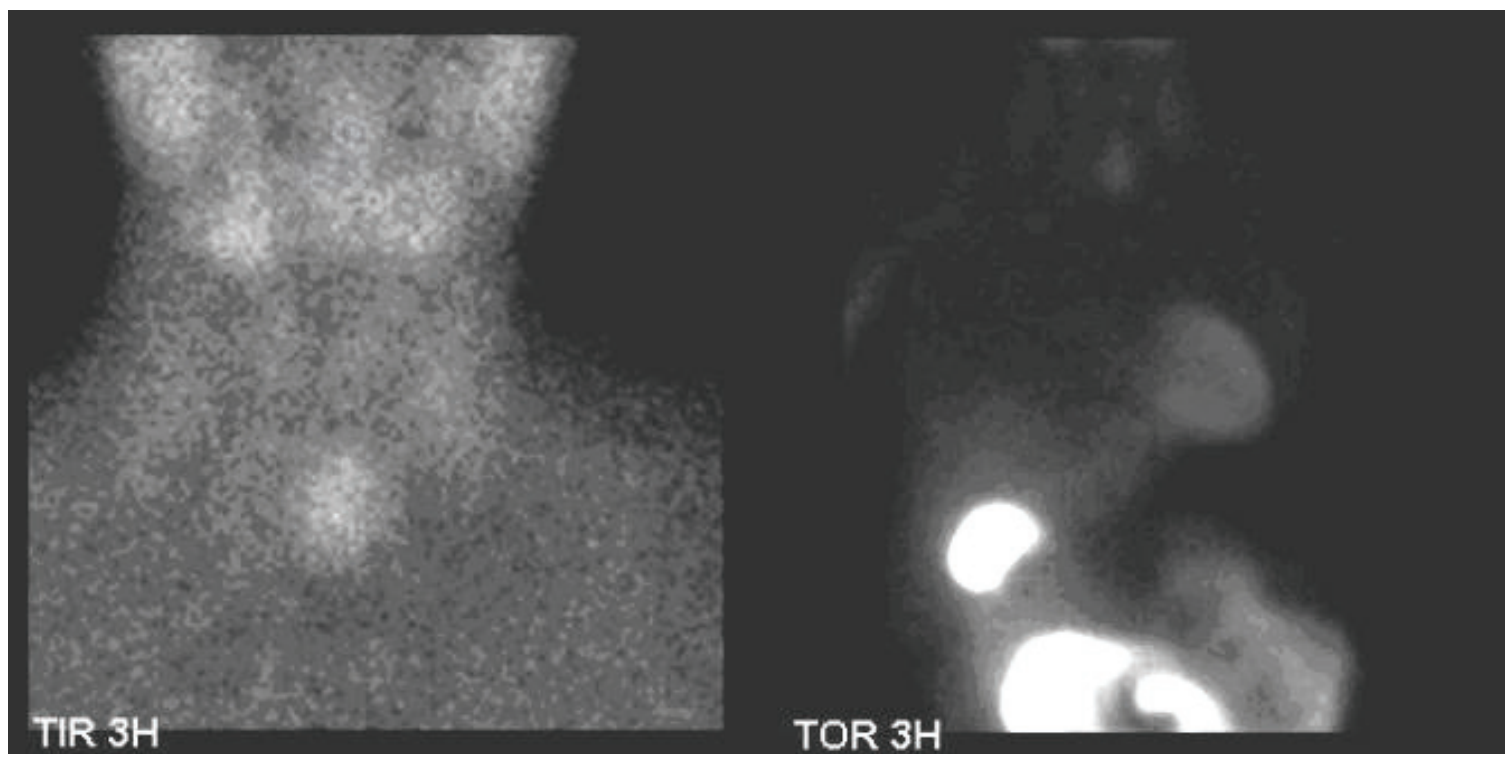

Figure 3. Parathyroid scintigraphy. SestaMIBI is a monovalent lipophilic cation that diffuses passively across cell membranes, concentrates in mitochondria and accumulates in adenomatous parathyroid tissue because of increased blood supply, higher metabolic activity and the absence of glycoprotein on the cell membrane [2]. Parathyroid scintigraphy was performed immediately after thyroid scintigraphy and the patient was given $740 \mathrm{MBq}$ of Tc ${ }^{99 \mathrm{~m}}$-sestaMIBI followed by 600 $\mathrm{mg}$ of sodium perchlorate for thyroid blocking. Additional anterior images of the neck were acquired 20 minutes and 3 hours after, followed then by a late chest image. All the images were obtained with a Millennium VG gamma camera (GE Healthcare, Wisconsin, USA) using a LEHR collimator. Parathyroid scintigraphy using sestaMIBI showed an intense and persistent uptake on the tracer in the left lower left pole of the thyroid. No other focus of uptake was found in the chest image.

technique in 6,331 cases gave values of $90.7 \%$ and $98.8 \%$, respectively [4]. The sensitivity of $\mathrm{Tc}^{99 \mathrm{~m}}$-sestaMIBI is limited in patients affected by parathyroid multiglandular disease and multinodular goiter.

As we demonstrated in this case, the parathyroid adenoma could present an intense uptake of pertechnetate, that

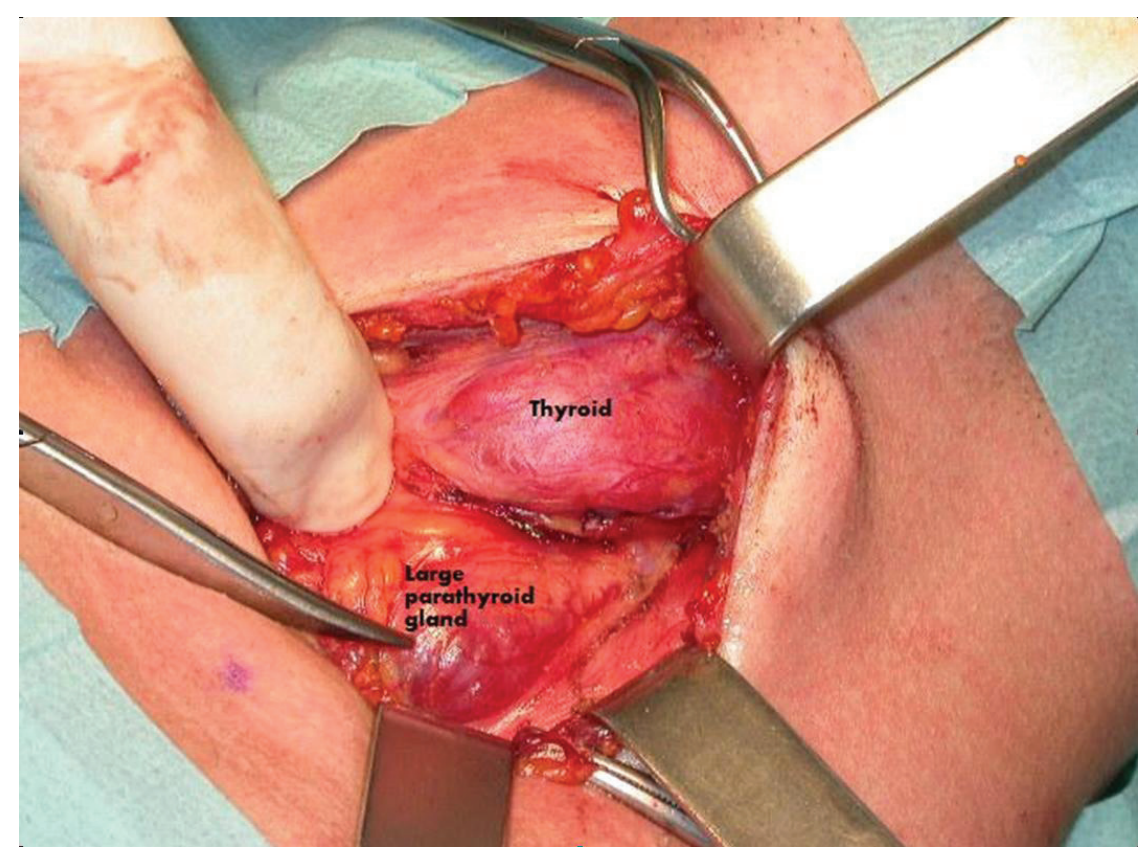

Figure 4. Surgical procedure. During surgery a large parathyroid gland was identified near the left thyroid lobe. 


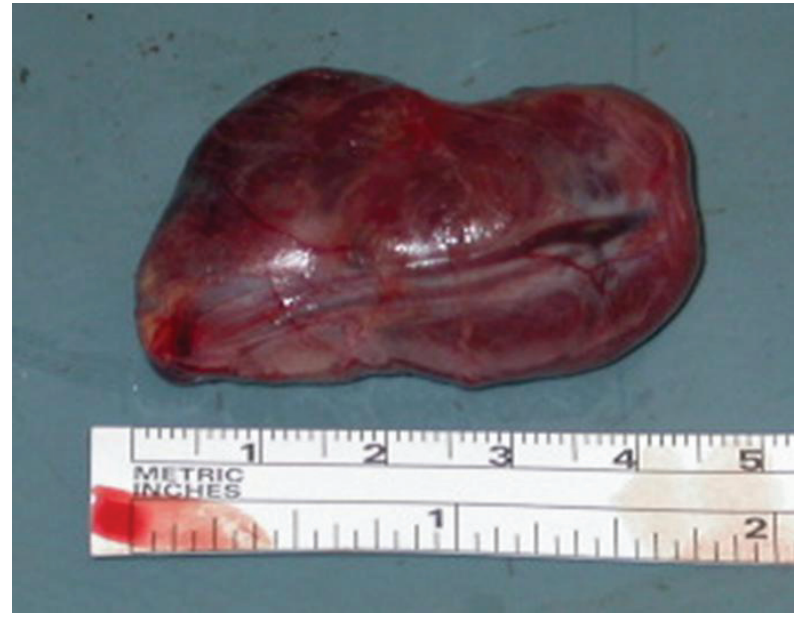

Figure 5. Excised parathyroid gland. The large $(45 \times 30 \times 25$ $\mathrm{mm}$ ) superior left parathyroid gland was excised and afterwards diagnosed as an adenoma.

is very unusual. Particularly in patients with multinodular goiter, the focal uptake of $\mathrm{Tc}^{99 \mathrm{~m}}$-perthecnetate and $\mathrm{Tc}^{99 \mathrm{~m}}$-sestaMIBI could be therefore attributed to a hyper functioning thyroid node rather than, as we demonstrated to be the case, a parathyroid adenoma.

\section{Consent}

Written informed consent was obtained from the patient for publication of this case report and accompanying images. A copy of the written consent is available for review by the Editor-in-Chief of this journal.

\section{Competing Interests}

The authors declare that they have no competing interests.

\section{Authors' Contributions}

AF and FA performed ecography and scintigraphy. IE per-

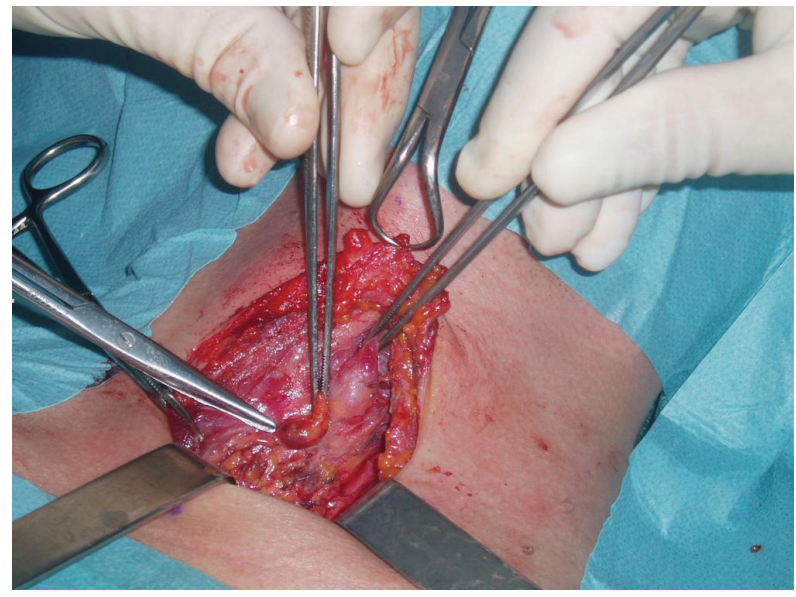

Figure 6. Normal parathyroid gland. The lower left parathyroid gland was grossly normal and therefore was not excised also because PHT decreased abruptly during surgical intervention.

formed the surgical procedure. GB admitted and follows up the patient. GF performed the hystopathology. AF and SC wrote and reviewed the manuscript. All authors read and approved the final manuscript.

\section{References}

1. Mitchell BK, Cornelius EA, Zoghbi S, Murren JR, Ghoussoub R, Flynn SD, Kinder BK. Mechanism of technetium $99 \mathrm{~m}$ sestamibi parathyroid imaging and the possible role of p-glycoprotein. Surgery 1996;120(6):10391045 .

2. Siegel A, Mancuso M, Seltzer M. The spectrum of positive scan patterns in parathyroid scintigraphy. Clin Nucl Med 2007;32(10):770-774.

3. Denham DW, Norman J. Cost-effectiveness of preoperative sestamibi scan for primary hyperparathyroidism is dependent solely upon the surgeon's choice of operative procedure. J Am Coll Surg 1998;186(3):293-305.

4. Liu Y, Chun KJ, Freeman LM. 'Shine through' on dual tracer parathyroid scintigraphy: a potential pitfall in interpretation. Clin Nucl Med 2005;30(3):145-149. 